\title{
A Penalty Method for Nonlinear Programs with Set Exclusion Constraints
}

\author{
Ben Hermans ${ }^{a}$, Goele Pipeleers ${ }^{a}$, Panagiotis Patrinos ${ }^{b}$ \\ ${ }^{a}$ MECO Research Team, Department of Mechanical Engineering, KU Leuven, Leuven, Belgium \\ DMMS_M lab, Flanders Make, Leuven, Belgium. \\ ${ }^{\mathrm{b}}$ Division ESAT-STADIUS, Department of Electrical Engineering, KU Leuven, Leuven, Belgium
}

\begin{abstract}
A common requirement in optimal control problems arising in autonomous navigation is that the decision variables are constrained to be outside certain sets. Such set exclusion constraints represent obstacles that must be avoided by the motion system. This paper presents a simple and efficient method for solving optimization problems with general set exclusion and implicit constraints. The method embeds the set exclusion constraints in a quadratic penalty framework and solves the inner optimization problems using a proximal algorithm that deals directly with the implicit constraints. We derive convergence results for this method by transforming the generated iterates to points of a reformulated problem with complementarity constraints. Furthermore, the practical application of the solution method is validated in numerical simulations of a model predictive control approach to path planning for a mobile robot. Finally, a runtime comparison with state-of-the-art solvers applied to the problem with complementarity constraints illustrates the efficiency of the proposed method.
\end{abstract}

Key words: Obstacle avoidance, Optimization, Convergence results, Optimal control, Nonlinear programming, Complementarity problems

\section{Introduction}

This paper focuses on problems where an objective is minimized in the presence of set exclusion constraints. Such constraints exclude certain open sets from the feasible set. This type of constraint arises often in path planning for example, where the set exclusion constraints represent obstacles in space, which is why set exclusion constraints and obstacle constraints will be used as synonyms in this paper. Another example of set exclusion constraints are the forbidden zones in attitude control of satellites, arising due to light sensitive equipment that may not be pointed towards the sun and possibly other bright celestial objects. In path planning popular approaches to deal with such constraints is to discretize the domain excluding obstacles and perform a graph search or potential field methods that model an attractive force from the destination and repulsive forces originated by obstacles.

In terms of numerical optimization, set exclusion constraints are almost always nonconvex even if the sets themselves are convex, hence complicating the resulting problems. Furthermore, set exclusion constraints can only be incorporated straightforwardly in special cases. For example, if the exclu-

Email address: ben. hermans2@kuleuven. be (Ben Hermans). sion set is defined by one inequality, this constraint reduces to a regular inequality constraint, as is the case for circular obstacles [24]. In the case of convex obstacles and convex motion systems, the separating hyperplane theorem also provides a way to transform these set exclusion constraints into regular inequality constraints [13]. Another commonly used approach is to impose via inequality constraints that the distance to the (convex) exclusion sets should be positive or larger than a small positive margin [6]. The disadvantage of such an approach is that calculating the distance to a general convex set requires the solution of a minimization problem. Moreover, the distance function is only differentiable if the sets are strictly convex. A similar idea is to impose that the gauge function of every set is bounded below by 1 , since this function evaluated at a point represents the smallest scaling factor so that the point is included in the set. This approach is especially useful when the polar set of each obstacle is easily representable, such as for polytopic obstacles [14].

The approach presented in this paper is applicable to problems with general and possibly nonconvex set exclusion constraints. It is a generalization of an earlier work [8], in which the approach presented here was shown to efficiently solve optimal control problems (OCP) arising in path planning. The approach involves incorporating the nonsmooth set exclusion constraints in a quadratic penalty framework. In the literature, convergence of the quadratic penalty method and 
the augmented Lagrangian method applied to smooth problems is covered in the monograph of Bertsekas [3]. In [4, Chapter 6] convergence results are extended to problems with implicit set constraints on the decision variables. In particular, it is shown that every limit point is a stationary point of an infeasibility measure but no conditions guaranteeing feasibility of limit points are provided. In this paper, it is shown that the considered problem can be reformulated through the introduction of slack variables to a mathematical program with vertical complementarity constraints (MPCC), first introduced in [21]. Consideration of the reformulated problem is necessary in the derivations as the set exclusion constraints are in general nonsmooth, which complicates the formulation of optimality conditions. The nonsmooth formulation using the normal cone of the constraint set from [16] is furthermore not usable, as in general the normal cone, or an outer approximation of this set, cannot be derived from the functions defining the set exclusion constraints.

MPCCs are a special class of optimization problems that often arise in practice. The authors of [18] discuss different notions of stationarity for such problems. The relevant stationarity conditions are reproduced in this paper, with a minor generalization to account for implicit set constraints, which is obtained from [16]. Although such problems present theoretical difficulties, as no solution satisfies the MangasarianFromovitz constraint qualification, they can nevertheless be solved fairly reliably by standard nonlinear optimization solvers [11]. KNITRO [23] is an interior-point solver and has some special features to exploit the structure of complementarity constraints [12]. SQP methods with an elastic mode for dealing with infeasible constraints, such as SNOPT [7], have also been shown to converge locally for problems with complementarity constraints [2]. Finally, the application of augmented Lagrangian approaches to problems with complementarity constraints was investigated in [9].

\subsection{Contributions}

The main contribution of this paper consists of theoretical convergence results showing that the limit points of the sequence generated by the quadratic penalty method applied to the original problem with set exclusion constraints, if they exist, satisfy stationarity conditions of a reformulated problem with complementarity constraints. Depending on the assumption the limit point might satisfy different stationarity conditions. As far as the authors know, the theoretical results presented in this paper are not covered by the results in literature, as discussed above. Furthermore, in simulations the performance of the method is compared to state-of-theart solvers KNITRO, IPOPT [22] and SNOPT applied to the equivalent problem with complementarity constraints. This comparison shows that the method outperforms other solvers applied to the problem with complementarity constraints, and combined with an earlier comparison with state-of-theart methods applied to the original problem [8], establishes the overall efficiency of the proposed approach in dealing with problems with general set exclusion constraints.
The remainder of this paper is organized as follows: Section 2 presents the optimization problem considered in this paper including the mathematical formulation introduced in [17] and the solution approach of [8]. Furthermore, it introduces the reformulated problem with complementarity constraints and the relevant stationarity conditions. Section 3 presents and proves the main convergence results of this paper. Section 4 derives the path planning problem for a bicycle from the proposed formulation and presents the numerical simulation results. Finally, Section 5 concludes the paper.

\subsection{Notation}

For $x \in \mathbb{R}^{n_{x}}$, let $x_{i}$ denote the $i$-th component of $x$. We define also the operator $[x]_{+}=\max (x, 0)$. Let $\left\{x^{v}\right\}$ denote a sequence of vectors, and $x^{v}$ the $v$-th entry in this sequence. For a nonempty closed convex set $X \subseteq \mathbb{R}^{n_{x}}$, let $N_{X}(x)$ denote the normal cone to this set at $x, N_{X}(x)=\left\{v \in \mathbb{R}^{n_{x}}\right.$ : $\langle v, y-x\rangle \leq 0$ for all $y \in X\}$, see Theorem 6.9 in [16].

\section{Methodology}

This section defines the class of optimization problems considered in this paper and describes the mathematical formulation of obstacles first introduced by [17]. It also presents the definition of the obstacle cost and extended obstacle cost functions. Furthermore, two equivalent reformulations for the problem are presented, the first of which is used in the solution algorithm described in the second subsection. The second reformulation is the one with complementarity constraints, used in the convergence results in Section 3 and for which the relevant optimality conditions are presented here.

\subsection{Optimization problem}

This paper considers mathematical programs with set exclusion constraints of the following form:

$$
\begin{array}{ll}
\underset{x \in X}{\operatorname{minimize}} & \ell(x), \\
\text { subject to } & x \notin O_{i}, \quad i=1, \ldots, n,
\end{array}
$$

with $x \in \mathbb{R}^{n_{x}}$ the decision variable that is constrained to lie in some nonempty closed convex set $X$ and outside of some nonempty obstacle sets $O_{i}$. The objective function $\ell: \mathbb{R}^{n_{x}} \rightarrow \mathbb{R}$ is assumed to be continuously differentiable. Additional equality and inequality constraints are not considered here for the sake of brevity. However, the conditions in Section 2 and the theoretical analysis in Section 3 could be straightforwardly extended to take these into account. In this work, $O_{i}$ is given by a set of nonlinear inequalities:

$$
O_{i}=\left\{x \in \mathbb{R}^{n_{x}}: h_{i j}(x)>0, j=1, \ldots, m_{i}\right\} .
$$

Here, the functions $h_{i j}: \mathbb{R}^{n_{x}} \rightarrow \mathbb{R}$ are continuously differentiable functions describing obstacles boundaries. The obsta- 
cle boundary $\partial O_{i}$ and the closure $\bar{O}_{i}$ are given as:

$$
\begin{aligned}
\partial O_{i} & =\left\{x \in \mathbb{R}^{n_{x}}: h_{i j}(x)=0, h_{i k}(x) \geq 0,\right. \\
& \text { for some } \left.j \in\left\{1, \ldots, m_{i}\right\}, k \neq j\right\} . \\
\bar{O}_{i} & =O_{i} \cup \partial O_{i} .
\end{aligned}
$$

In general optimization problems, equality constraints and inequality constraints that hold as equality at a feasible point are called active, and inequality constraints that hold strictly at this point are called inactive. We will reuse this terminology and introduce the set of active obstacles $I_{\bar{O}}(x)$ and the set of active obstacle boundaries $I_{a}(x)$ :

$$
\begin{aligned}
I_{\bar{O}}(x) & =\left\{i: x \in \bar{O}_{i}\right\} . \\
I_{a}(x) & =\left\{(i, j): i \in I_{\bar{O}}(x), h_{i j}(x)=0\right\} .
\end{aligned}
$$

As shown in [17], we can encode each obstacle avoidance constraint as the following equality constraint:

$$
\psi_{i}(x):=\prod_{j=1}^{m_{i}}\left[h_{i j}(x)\right]_{+}=0 .
$$

The obstacle cost function $\psi_{i}(x)$ is by definition strictly positive for $x \in O_{i}$, and exactly zero outside $O_{i}$. Problem (1) can then be reformulated using the obstacle cost functions:

$$
\begin{array}{cl}
\underset{x \in X}{\operatorname{minimize}} & \ell(x), \\
\text { subject to } & \psi_{i}(x)=0, \quad i=1, \ldots, n .
\end{array}
$$

Note that each obstacle cost function $\psi_{i}$ is differentiable at points strictly inside and strictly outside the obstacle, but due to the presence of the $[.]_{+}$operators, it is not differentiable at points on the obstacle boundary. We will use also a smooth version of this function which can be constructed by dropping the $[.]_{+}$operators, resulting in the extended $o b$ stacle cost function $\widetilde{\psi}_{i}(x)$ and its gradient:

$$
\widetilde{\psi}_{i}(x)=\prod_{j=1}^{m_{i}} h_{i j}(x), \quad \nabla \widetilde{\psi}_{i}(x)=\sum_{j=1}^{m_{i}} \nabla h_{i j}(x) \prod_{k \neq j} h_{i k}(x) .
$$

\subsection{Penalty method}

The obstacle avoidance constraints will be dealt with using a quadratic penalty term that is added to the objective function [8]. The reformulated optimization problem is therefore

$$
\underset{x \in X}{\operatorname{minimize}} \mathscr{L}_{\mu}(x),
$$

with $\mathscr{L}_{\mu}(x)=\ell(x)+\frac{\mu}{2} \sum_{i=1}^{n} \psi_{i}^{2}(x)$. Here, $\mu$ is the penalty parameter corresponding to the obstacle avoidance constraints. Using a vector of penalty factors will not be considered in this paper for the sake of keeping the presentation simple.
Note that $\mathscr{L}_{\mu}(x)$ is continuously differentiable, with gradient

$$
\begin{aligned}
\nabla \mathscr{L}_{\mu}(x) & =\nabla \ell(x)+\mu \sum_{i=1}^{n} \sum_{j=1}^{m_{i}} \nabla h_{i j}(x)\left[h_{i j}(x)\right]_{+} \prod_{k \neq j}\left[h_{i k}(x)\right]_{+}^{2} \\
& =\nabla \ell(x)+\mu \sum_{i=1}^{n} \sum_{j=1}^{m_{i}} \nabla h_{i j}(x) \psi_{i}(x) \prod_{k \neq j} h_{i k}(x) \\
& =\nabla \ell(x)+\mu \sum_{i=1}^{n} \psi_{i}(x) \nabla \widetilde{\psi}_{i}(x) .
\end{aligned}
$$

In this derivation we used $\nabla\left([w]_{+}^{2}\right)=2[w]_{+} \nabla w$ and $[w]_{+}^{2}=$ $[w]_{+} w$. Solving each problem (6) for a fixed value $\mu^{v}$ is performed approximately, yielding $x^{v} \in X$ as an $\varepsilon^{v}$-approximate KKT point, that is, a feasible point $x^{v}$ satisfying

$$
\exists e^{v}: e^{v} \in \nabla \mathscr{L}_{\mu^{v}}\left(x^{v}\right)+N_{X}\left(x^{v}\right), \quad\left\|e^{v}\right\| \leq \varepsilon^{v} .
$$

Here, $\left\{\varepsilon^{v}\right\}$ is a sequence of positive tolerances tending towards zero as $v \rightarrow \infty$. The steps are given in Algorithm 1 .

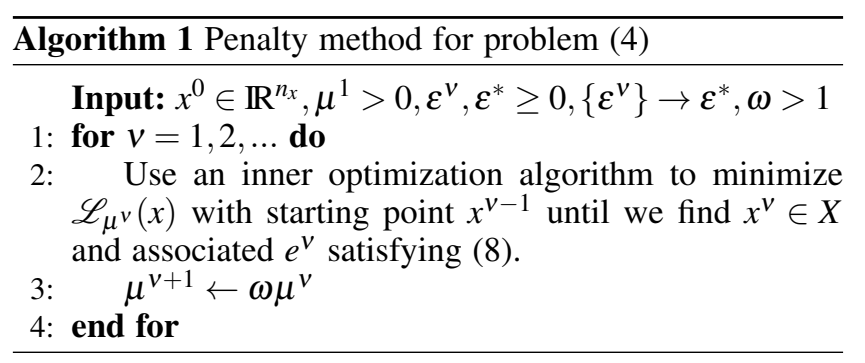

Remark 1 In Algorithm 1, no termination criteria are provided. In practice of course, the algorithm would either terminate when $\varepsilon^{v}=\varepsilon^{*}$ and $\forall i: \psi_{i}\left(x^{v}\right) \leq \eta^{*}$, with $\varepsilon^{*}$ and $\eta^{*}$ some small positive tolerances, or if it gets stuck in a point of local infeasibility.

\section{$2.3 M P C C$}

A mathematical program with vertical complementarity constraints can be written in the following form [18].

$$
\begin{aligned}
\underset{z \in Z}{\operatorname{minimize}} & f(z), \\
\text { subject to } & G(z) \leq 0, \\
& \min \left\{F_{i 1}(z), \ldots, F_{i l}(z)\right\}=0, \quad i=1, \ldots, m,
\end{aligned}
$$

where $z \in \mathbb{R}^{n_{z}}, f: \mathbb{R}^{n_{z}} \rightarrow \mathbb{R}, G: \mathbb{R}^{n_{z}} \rightarrow \mathbb{R}^{p}, F: \mathbb{R}^{n_{z}} \rightarrow \mathbb{R}^{m \times l}$. The implicit constraints $z \in Z$, with $Z$ some nonempty closed convex set, are here added and equality constraints are left out from the formulation used in [18]. The vertical complementarity constraints $\min \left\{F_{i 1}(z), \ldots, F_{i l}(z)\right\}=0$ are a generalization of regular complementarity constraints which only allow for two vectors, that is $\min \left\{F_{i 1}(z), F_{i 2}(z)\right\}=0$. By introducing slack variables $t_{i j}$, problem (4) can be reformulated as a problem of type (9).

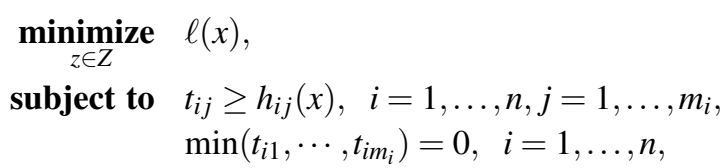


with $z=(x, t), Z=X \times \mathbb{R}^{n_{t}}$ and $n_{t}=\sum_{i=1}^{n} m_{i}$. This problem is indeed of type (9), with smooth functions $f(z)=\ell(x)$, $G_{k}(z)=h_{i j}(x)-t_{i j}$, with $k=j+\sum_{l=1}^{i-1} m_{l}$, and $F_{i j}(z)=t_{i j}$. We will next show that problems (4) and (10) are equivalent. Firstly, they share the same cost function which is independent of $t$. Next, assume $(x, t)$ is feasible for (10). Then $x$ is obviously feasible for (4). On the other hand, assume that $x$ is feasible for (4) and let

$t_{i j}= \begin{cases}h_{i j}(x), & i \in I_{\bar{O}}(x), \\ 0, & i \notin I_{\bar{O}}(x), j=\min _{\operatorname{argmin}}\left\{h_{i k}(x)\right\}, \\ {\left[h_{i j}(x)\right]_{+}+\varepsilon,} & i \notin I_{\bar{O}}(x), j \neq \min _{\operatorname{argmin}}\left\{h_{i k}(x)\right\},\end{cases}$

with $\varepsilon>0$. Then, Remarks 2 and 3 show that the resulting point $(x, t)$ is feasible for problem (10).

Remark 2 From (2) we have that for an obstacle $i$ to be inactive, at least one of the boundary functions has to be strictly negative, that is $\exists j: h_{i j}(x)<0$. For such $j$, the slack variable can be set to zero to satisfy the constraints in (10). In the third case of (11), the slack variables can be chosen freely as long as they are strictly greater than $h_{i j}(x)$ and 0 . This is necessary to prevent obstacle boundaries $(i, j): i \notin$ $I_{\bar{O}}, h_{i j}(x)=0$ yielding active constraints in problem (10).

Remark 3 If $\psi_{i}(x)=0$, and $t$ is given by (11), then the corresponding complementarity condition for $(x, t)$ in problem (10) is also satisfied. This follows from the fact that either $i \notin I_{\bar{O}}(x)$ and the second case of (11) applies, or $i \in I_{\bar{O}}(x)$ and therefore there is at least one jor which $h_{i j}(x)=t_{i j}=0$, according to the first case of (11).

\subsection{Optimality conditions}

In this subsection, the definitions of the linear independence constraint qualification (LICQ) for MPCCs (MPCC-LICQ), the strong stationarity conditions and the Clarke stationarity conditions are presented and applied to problem (10). Furthermore, two assumptions that will be used in the convergence results are shown to imply MPCC-LICQ.

\subsubsection{Constraint qualifications}

Applying the MPCC-LICQ from [18] to problem (9), we obtain the condition that there exists no vector $y=\left(y^{G}, y^{F}\right) \neq$ 0 that satisfies

$$
-\sum_{r: G_{r}(z)=0} y_{r}^{G} \nabla G_{r}(z)-\sum_{(i, j): F_{i j}(z)=0} y_{i j}^{F} \nabla F_{i j}(z) \in N_{Z}(z) .
$$

To translate this condition for problem (10), let the index set $I_{h}(z)$ and $I_{t}(z)$ denote respectively the active inequality constraints and the active complementarity components at $z$ :

$$
\begin{aligned}
I_{h}(z) & =\left\{(i, j): h_{i j}(x)-t_{i j}=0\right\}, \\
I_{t}(z) & =\left\{(i, j): t_{i j}=0\right\} .
\end{aligned}
$$

Furthermore, from Proposition 6.41 in [16], we have that $N_{Z}(z)=N_{X}(x) \times 0$. Note that we omit the dependence of the index sets on $z$ in the following definition for the sake of brevity in the mathematical expressions.

Definition 4 A feasible point $z=(x, t)$ of problem (10) satisfies MPCC-LICQ iff there exists no vector $\left(y^{h}, y^{t}\right) \neq 0$ satisfying the following conditions

$$
\left\{\begin{array}{l}
y_{i j}^{h}=0 \text { for }(i, j) \in I_{h} \backslash I_{t}, \\
y_{i j}^{t}=0 \text { for }(i, j) \in I_{t} \backslash I_{h}, \\
-\sum_{(i, j) \in I_{h} \cap I_{t}} y_{i j}^{h} \nabla h_{i j}(x) \in N_{X}(x) .
\end{array}\right.
$$

Remark 5 It is well known that LICQ implies the regular and strict Mangasarian-Fromovitz constraint qualifications (MCFQ and SMFCQ), [10, Chapter 2]. This also holds for the constraint qualifications defined for MPCCs. Therefore, only MPCC-LICQ will be used in this paper. However, as shown in [19], this is not a stringent assumption for MPCCs.

In proofs of the convergence results (cf. Theorems 13 and 14), assumptions will be used to prove that limit points of the generated sequences, if they exist, satisfy certain stationarity conditions. These assumptions can be thought of as mirroring linear independence of the active constraint gradients for the obstacle formulations. Before we introduce these assumptions, we need the following result:

Lemma 6 Assume that $(x, t)$ is feasible for (10) and $t$ is given by (11). Then

$$
I_{a}(x)=I_{h}(x, t) \cap I_{t}(x, t) .
$$

PROOF. This lemma can be proven by showing the set inclusion in both directions. In this proof the dependence of the index sets on the variables will be omitted. From (3) and the first case of (11) it follows that for $(i, j) \in I_{a}: h_{i j}(x)=$ $t_{i j}=0$. Therefore, we immediately have $I_{a} \subset I_{h} \cap I_{t}$.

The other way around, suppose that $(i, j) \in\left(I_{h} \cap I_{t}\right) \backslash I_{a}$. From $(i, j) \in I_{h} \cap I_{t}$ we have that $h_{i j}(x)=t_{i j}=0$. Then, from $(i, j) \notin I_{a}$ and (3), it follows that $i \notin I_{\bar{O}}$. However, this implies that $(i, j)$ does not belong to the first case of (11). Neither does it belong to the second case as $h_{i j}(x)=0$, cf. Remark 2. Therefore $(i, j)$ belongs to the third case of (11), and thus $t_{i j}=\varepsilon>0$. This disproves the assumption that $(i, j) \in\left(I_{h} \cap I_{t}\right)$, and therefore proves that $\left(I_{h} \cap I_{t}\right) \subset I_{a}$.

The following lemmas prove that certain assumption imply the conditions (15).

Lemma 7 Assume that at a point $x$, there is no vector $y$ satisfying

$$
-\sum_{i \in I_{\bar{O}}(x)} y_{i} \nabla \widetilde{\psi}_{i}(x) \notin N_{X}(x) \text { unless } y=0 .
$$

If, furthermore, $(x, t)$ is feasible for problem (10), with $t$ given by (11), then (15) is satisfied at this point. 
PROOF. Note that because $0 \in N_{X}(x)$, (16) implies that the active obstacle cost gradients are linearly independent and thus not equal to zero. Given (5), this excludes the case where there is more than one active obstacle boundary. Moreover, because $x$ is feasible, $x \notin O_{i}$, and thus for $i \in I_{\bar{O}}(x): x \in$ $\bar{O}_{i} \backslash O_{i}=\partial O_{i}$, which implies that for each active obstacle, there is at least one active obstacle boundary. In conclusion, for each active obstacle, there is exactly one active obstacle boundary. Let $j_{i}$ denote the index of this active boundary, such that $h_{i j_{i}}(x)=0$. From (5) it then follows that

$$
\nabla \widetilde{\psi}_{i}(x)=\nabla h_{i j_{i}}(x) \prod_{k \neq j_{i}} h_{i k}(x), \quad\left(i, j_{i}\right) \in I_{a}(x) .
$$

From (17) and Lemma 6, (16) is now equivalent to (15).

As mentioned in the proof of Lemma 7, equation (16) can only be satisfied if for every active obstacle, there is only one active obstacle boundary and the gradient of this boundary function is nonzero at its boundary. Boundary functions with this latter property must therefore be chosen in order for the algorithm to work provably. We provide also an assumption that covers the case of multiple active boundaries.

Lemma 8 If at a feasible point $(x, t)$, with $t$ given by (11), there is no vector y satisfying

$$
-\sum_{(i, j) \in I_{a}(x)} y_{i j} \nabla h_{i j}(x) \notin N_{X}(x) \text { unless } y=0,
$$

then (15) is satisfied at this point.

PROOF. The proof for this lemma is immediate as according to Lemma 6, (18) and (15) are equivalent.

Remark 9 The conditions (18) and (15) are only equivalent because of our choice of the slack variables (11). In case we had chosen $\varepsilon=0$ in (11), (15) would be a set of stricter conditions than (18). However, as we neglect these points, (18) and (15) can be considered as equivalent. Note also that if (16) holds at a feasible point then (18) holds at this point because of (17).

\subsubsection{Stationarity conditions}

For mathematical programs with vertical complementarity constraints, different notions of stationarity exist, such as the strong and Clarke stationarity conditions. In order to introduce these conditions for problem (9), we first introduce the Lagrangian $L$ associated with this problem [18], with $\alpha=1$.

$$
L(z, \Gamma, \lambda)=f(z)-\sum_{i=1}^{m} \sum_{j=1}^{l} F_{i j}(z) \Gamma_{i j}+\sum_{i=1}^{p} G_{i}(z) \lambda_{i} .
$$

The first set of stationarity conditions we derive are the strong stationarity condtions. By applying Corollary 6.15 of [16] to a relaxed NLP (RNLP) formulation of the MPCC [18], the strong stationarity conditions are given as follows.
Definition 10 For a feasible point $z$ of problem (9) the strong stationarity conditions are the following conditions on the point $z$ and the multipliers $\Gamma$ and $\lambda$

$$
\begin{aligned}
0 & \in \nabla_{z} L(z, \Gamma, \lambda)+N_{Z}(z), \\
F_{i j}(z) \Gamma_{i j} & =0, \quad i=1, \ldots, m, j=1, \ldots, l, \\
\lambda_{i} & \geq 0, \quad i=1, \ldots, p, \\
G_{i}(z) \lambda_{i} & =0, \quad i=1, \ldots, p, \\
\Gamma_{i j} & \geq 0, \quad \text { if } \exists k \neq j: t_{i j}=t_{i k}=0 .
\end{aligned}
$$

The strong stationarity conditions are only necessary in the general case when strict complementarity holds [18], stating that for a point $z$ of problem (9), there is for every $i$ only one $j_{i}$ such that $F_{i j_{i}}(z)=0$. From Defintion 10, with (19a) split according to $x$ and $t$, we can now to derive the strong stationarity conditions for problem (10) as the following conditions on $(x, t)$ and the unique multipliers $(\Gamma, \lambda)$

$$
\begin{aligned}
0 \in \nabla \ell(x)+\sum_{i=1}^{n} \sum_{j=1}^{m_{i}} \lambda_{i j} \nabla h_{i j}(x)+N_{X}(x), \\
\lambda_{i j}+\Gamma_{i j}=0, \quad i=1, \ldots, n, j=1, \ldots, m_{i}, \\
t_{i j} \Gamma_{i j}=0, \quad i=1, \ldots, n, j=1, \ldots, m_{i}, \\
\lambda_{i j} \geq 0, \quad i=1, \ldots, n, j=1, \ldots, m_{i}, \\
\left(h_{i j}(x)-t_{i j}\right) \lambda_{i j}=0, \quad i=1, \ldots, n, j=1, \ldots, m_{i}, \\
\Gamma_{i j} \geq 0, \quad \text { if } \exists k \neq j: t_{i j}=t_{i k}=0,
\end{aligned}
$$

Another set of stationarity conditions used in this paper are the Clarke stationarity conditions.

Theorem 11 If for a local minimizer $z$ of problem (9) there is no vector $\left(y^{G}, y^{F}\right) \neq 0$ for which (12) holds, i.e MPCCLICQ holds, then there exist multipliers $\Gamma$ and $\lambda$ such that

$$
\begin{aligned}
0 & \in \nabla_{z} L(z, \Gamma, \lambda)+N_{Z}(z), \\
F_{i j}(z) \Gamma_{i j} & =0, \quad i=1, \ldots, m, j=1, \ldots, l, \\
\lambda_{i} & \geq 0, \quad i=1, \ldots, p, \\
G_{i}(z) \lambda_{i} & =0, \quad i=1, \ldots, p, \\
\Gamma_{i j} \Gamma_{i k} & \geq 0, \quad(j, k): F_{i j}(z)=F_{i k}(z)=0 .
\end{aligned}
$$

PROOF. The proof here is completely analogous to the proof of Theorem 2 in [18], but without neglecting the normal cone contribution in Theorem 1 of [5].

The conditions (21a) - (21e) are the Clarke stationarity conditions. Clearly, they do not differ from the strong stationarity conditions (19a) - (19e), aside from (19e) which is now replaced by $(21 \mathrm{e})$. Hence, the Clarke conditions for problem (10) are the conditions (20a) - (20e), together with

$$
\Gamma_{i j} \Gamma_{i k} \geq 0, \quad(j, k): t_{i j}=t_{i k}=0 .
$$

Example 3 in [18] illustrates a point satisfying the Clarke but not the strong stationarity conditions. 


\section{Convergence results}

This section proves that if the sequence of iterates found by Algorithm 1 for increasing values of the penalty parameter has limit points, then these satisfy either the strong or the Clarke stationarity conditions of problem (10), depending on the assumptions made. The first part of each proof is similar to the proof of Proposition 2.3 in [3]. The second part derives the remaining conditions from the definitions of the slack and multiplier variables.

Remark 12 If the set $X$ is compact, then the sequence has limit points. This condition is satisfied for instance in the simulation example of Section 4. However, we do not wish to restrict the theoretical results here to this special case.

Theorem 13 Let $\left\{x^{v}\right\}$ be a sequence of iterates generated by Algorithm 1, with $\varepsilon^{*}=0$. Assume that a subsequence $\left\{x^{v}\right\}_{v \in K}$ converges to a vector $x^{*}$ such that (16) holds at $x^{*}$, with the set of slack variables $t^{*}$ defined as in (11). Then, point $\left(x^{*}, t^{*}\right)$ is feasible for (10) and unique multipliers can be derived so that $\left(x^{*}, t^{*}, \lambda^{*}, \Gamma^{*}\right)$ satisfies the strong stationarity conditions (20a)-(20f) of problem (10).

PROOF. Most of the constraints of problem (10) are straightforwardly satisfied in point $\left(x^{*}, t^{*}\right)$. Line 2 in Algorithm 1 gives us $x^{v} \in X$, and thus because $X$ is closed also $x^{*} \in X$. Moreover, by construction, $t_{i j}^{*} \geq h_{i j}\left(x^{*}\right)$ is satisfied. The only constraints remaining to be shown are the complementarity conditions $\min \left(t_{i 1}, \ldots, t_{i m_{i}}\right)=0, i=1, \ldots, n$. This corresponds to showing that for each $i, \psi_{i}\left(x^{v}\right) \rightarrow 0$, cf. Remark 3. Define for all $v$

$$
\lambda_{i}^{v}=\mu^{v} \psi_{i}\left(x^{v}\right) .
$$

We have from (7) that

$$
\begin{aligned}
\nabla \mathscr{L}_{\mu^{v}}\left(x^{v}\right) & =\nabla \ell\left(x^{v}\right)+\sum_{i=1}^{n} \mu^{v} \psi_{i}\left(x^{v}\right) \nabla \widetilde{\psi}_{i}\left(x^{v}\right) \\
& =\nabla \ell\left(x^{v}\right)+\sum_{i=1}^{n} \lambda_{i}^{v} \nabla \widetilde{\psi}_{i}\left(x^{v}\right) \\
& =\nabla \ell\left(x^{v}\right)+\sum_{i \in I_{\bar{O}}\left(x^{v}\right)} \lambda_{i}^{v} \nabla \widetilde{\psi}_{i}\left(x^{v}\right) .
\end{aligned}
$$

For the last equality, note that for $i \notin I_{\bar{O}}\left(x^{v}\right), \psi_{i}\left(x^{v}\right)=0$ and thus $\lambda_{i}^{v}=0$. Furthermore, there exists some $v_{1} \in K$, such that for $K \ni v \geq v_{1}, x^{v}$ will be close enough to $x^{*}$ and therefore inactive obstacles at $x^{*}$ will also be inactive at $x^{v}$. Thus, for $K \ni v \geq v_{1}, I \frac{c}{O}\left(x^{*}\right) \subset I \frac{c}{O}\left(x^{v}\right)$, and by applying the complement we obtain $I_{\bar{O}}\left(x^{v}\right) \subset I_{\bar{O}}\left(x^{*}\right)$. Equation (23) for $K \ni v \geq v_{1}$ then becomes

$$
\nabla \mathscr{L}_{\mu^{v}}\left(x^{v}\right)=\nabla \ell\left(x^{v}\right)+\sum_{i \in I_{\bar{O}}\left(x^{*}\right)} \lambda_{i}^{v} \nabla \widetilde{\psi}_{i}\left(x^{v}\right) .
$$

Substituting (24) in (8) then yields for $K \ni v \geq v_{1}$ :

$$
e^{v} \in \nabla \ell\left(x^{v}\right)+\sum_{i \in I_{\bar{O}}\left(x^{*}\right)} \lambda_{i}^{v} \nabla \widetilde{\psi}_{i}\left(x^{v}\right)+N_{X}\left(x^{v}\right) .
$$

Assuming $\left\{x^{v}\right\}$ is a bounded sequence, then $\left\{\nabla \ell\left(x^{v}\right)\right\}$ is also bounded. Given that $e^{v}$ is also bounded explicitly by (8), this results in boundedness of $\sum_{i \in I_{\bar{O}}\left(x^{*}\right)} \lambda_{i}^{v} \nabla \widetilde{\psi}_{i}\left(x^{v}\right)$ for large enough $v \in K$. Indeed, if this sequence were not bounded, then the terms $e^{v}$ and $\nabla \ell\left(x^{v}\right)$ would become negligible in (25), which would then in the limit contradict (16). Furthermore, (16) also implies that for large enough $v \in K$, the gradients $\nabla \widetilde{\psi}_{i}\left(x^{v}\right), i \in I_{\bar{O}}\left(x^{*}\right)$ are linearly independent, thus we can solve (25) for the active multipliers

$$
\left.\lambda^{v}=\left[\nabla \widetilde{\psi}\left(x^{v}\right)^{\top} \nabla \widetilde{\psi}\left(x^{v}\right)\right]^{-1} \nabla \widetilde{\psi}\left(x^{v}\right)^{\top}\left(e^{v}-\nabla \ell\left(x^{v}\right)-w^{v}\right)\right),
$$

with $w^{v} \in N_{X}\left(x^{v}\right)$ satisfying (25). As $e^{v} \rightarrow 0$, it follows that $\left\{\lambda^{v}\right\}_{K} \rightarrow \bar{\lambda}=-\left[\nabla \widetilde{\psi}\left(x^{*}\right)^{\top} \nabla \widetilde{\psi}\left(x^{*}\right)\right]^{-1} \nabla \widetilde{\psi}\left(x^{*}\right)^{\top}\left(\nabla \ell\left(x^{*}\right)+w^{*}\right)$.

Hence, all $\bar{\lambda}_{i}$ have to be bounded and consequently, recalling (22) and because $\mu^{v} \rightarrow \infty$, all $\psi_{i}\left(x^{*}\right)$ must be equal to 0 . Therefore, the point $\left(x^{*}, t^{*}\right)$ is feasible for problem (10). Moreover, (25) can be written in the limit as

$$
0 \in \nabla \ell\left(x^{*}\right)+\sum_{i \in I_{\bar{O}}\left(x^{*}\right)} \bar{\lambda}_{i} \nabla \widetilde{\psi}_{i}\left(x^{*}\right)+N_{X}\left(x^{*}\right) .
$$

As (16) holds in $x^{*}$, it follows from Lemma 7 that the point $\left(x^{*}, t^{*}\right)$ satisfies MPCC-LICQ. Furthermore, from (17) and (11), strict complementarity holds at this point. Next, we show that the strong stationarity conditions are satisfied in this point. The multipliers for the reformulated problem can be retrieved as follows:

$$
\begin{aligned}
& \lambda_{i j}^{*}=\bar{\lambda}_{i} \prod_{k \neq j}\left[h_{i k}\left(x^{*}\right)\right]_{+}, \\
& \Gamma_{i j}^{*}=-\lambda_{i j}^{*}=-\bar{\lambda}_{i} \prod_{k \neq j}\left[h_{i k}\left(x^{*}\right)\right]_{+} .
\end{aligned}
$$

Consider from the discussion above that for the inactive obstacles $i \notin I_{\bar{O}}\left(x^{*}\right)$, the multipliers $\bar{\lambda}_{i}=0$. Thus, from the definition of the multipliers (27) and (28) it follows that

$$
\lambda_{i j}^{*}=\Gamma_{i j}^{*}=0, \quad i \notin I_{\bar{O}}\left(x^{*}\right) .
$$

Furthermore, as shown in the proof of Lemma 7, for each active obstacle there is exactly one active obstacle boundary. Let $j_{i}$ denote the index for which for each $i \in I_{\bar{O}}\left(x^{*}\right)$, $h_{i j_{i}}\left(x^{*}\right)=0$. Then, from (27), for $k \neq j_{i}$,

$$
\lambda_{i k}^{*}=\lambda_{i j_{i}}^{*} \frac{\left[h_{i j_{i}}\left(x^{*}\right)\right]_{+}}{\left[h_{i k}\left(x^{*}\right)\right]_{+}}=0, \quad(i, k) \notin I_{a}\left(x^{*}\right) .
$$

Equations (17), (26), (27), (29) and (30) combined show that (20a) is satisfied at $x^{*}$. The remaining strong stationarity conditions follow from the definition of the multipliers (27), (28) and the slack variables (11). By choice of the multipliers $\Gamma_{i j}^{*}$ in (28), (20b) is trivially satisfied. Considering (29), condition (20c) is trivially satisfied for $i \notin I_{\bar{O}}\left(x^{*}\right)$. For $i \in I_{\bar{O}}\left(x^{*}\right)$, as $t_{i j}^{*}=h_{i j}\left(x^{*}\right)$ and using (28) and (27), condition (20c) is transformed to

$$
t_{i j}^{*} \Gamma_{i j}^{*}=-\bar{\lambda}_{i} \psi_{i}\left(x^{*}\right), \quad i \in I_{\bar{O}}\left(x^{*}\right) .
$$

As shown above, $\psi_{i}\left(x^{*}\right)=0$ for all $i$, thus condition (20c) 
is satisfied. By construction of the multipliers $\lambda_{i j}^{*}$ in (27) and given $\bar{\lambda}_{i}$ is positive due to (22), condition (20d) is also satisfied. Condition (20e) is trivially satisfied for $i \notin I_{\bar{O}}\left(x^{*}\right)$ because of (29). For $i \in I_{\bar{O}}\left(x^{*}\right), t_{i j}^{*}=h_{i j}\left(x^{*}\right)$ and (20e) is thus also trivially satisfied for this case. Finally, condition (20f) does not apply in this case as strict complementarity holds because of (17) and definition (11).

Limit points where more than one boundary of the same obstacle is active are excluded by the assumption used in Theorem 13. The measure of this set relative to the entire boundary $\partial O$ is zero however, as its dimension is lower. Moreover, in case of a limit point where more than one obstacle boundary of the same obstacle is active, it can still be shown that this point satisfies the Clarke conditions (20a)(20e) and $(20 \mathrm{~g})$ under the assumption that this point is feasible and (18) holds. Note that in this case strict complementarity does not (necessarily) hold, as multiple active obstacle boundaries translate into multiple indices $j$ for which $t_{i j}=0$, according to the first case of (11).

Theorem 14 Let $\left\{x^{v}\right\}$ be a sequence of iterates generated by Algorithm 1, with $\varepsilon^{*}=0$. Assume that a subsequence $\left\{x^{v}\right\}_{v \in K}$ converges to a vector $x^{*}$ feasible for problem (4), such that (18) holds at $x^{*}$. Define the set of slack variables $t^{*}$ as in (11). Then, point $\left(x^{*}, t^{*}\right)$ satisfies the Clarke stationarity conditions, (20a)-(20e) and (20g) of problem (10).

PROOF. Given the point $x^{*}$ is feasible for problem (4), it follows that $\forall i: \psi_{i}\left(x^{*}\right)=0$, which implies feasibility of $\left(x^{*}, t^{*}\right)$ for problem (10), cf. Remark 3 , and also that for every $i$, there is at least one index $j$ for which $\left[h_{i j}\left(x^{*}\right)\right]_{+}=0$. Define for all $v$ :

$$
\begin{aligned}
& \lambda_{i}^{v}=\mu^{v} \psi_{i}\left(x^{v}\right) \\
& \lambda_{i j}^{v}=\lambda_{i}^{v} \prod_{l \neq j}\left[h_{i l}\left(x^{v}\right)\right]_{+} .
\end{aligned}
$$

Along the same reasoning as in the proof of Theorem 13, there exists some $v_{1} \in K$, such that for $K \ni v \geq v_{1}, I_{\bar{O}}\left(x^{v}\right) \subset$ $I_{\bar{O}}\left(x^{*}\right)$. The multipliers belonging to inactive obstacles will then be equal to zero for $K \ni v \geq v_{1}$. Furthermore, in the limit, the multipliers for the inactive obstacle boundaries will become negligible against the multipliers for the active ones. Let $j$ denote an index for which $\left[h_{i j}\left(x^{*}\right)\right]_{+}=0$, and $l$ an index for which $\left[h_{i l}\left(x^{*}\right)\right]_{+}>0$. Then

$$
\lim _{v \rightarrow \infty} \frac{\lambda_{i l}^{v}}{\lambda_{i j}^{v}}=\lim _{v \rightarrow \infty} \frac{\left[h_{i j}\left(x^{v}\right)\right]_{+}}{\left[h_{i l}\left(x^{v}\right)\right]_{+}}=0 .
$$

Assumption (18) implies that the gradients $\nabla h_{i j}\left(x^{*}\right),(i, j) \in$ $I_{a}\left(x^{*}\right)$ are linearly independent and thus different from the zero vector. Then, as a result of (31), terms in (25) with $(i, j) \notin I_{a}\left(x^{*}\right)$ will become negligible for large $v \in K$. Thus, (25) reduces in this case to

$$
e^{v} \in \nabla \ell\left(x^{v}\right)+\sum_{(i, j) \in I_{a}\left(x^{*}\right)} \lambda_{i j}^{v} \nabla h_{i j}\left(x^{v}\right)+N_{X}\left(x^{v}\right) .
$$

Since $e^{v} \rightarrow 0$, it follows that we can solve for the active multipliers and take the limit

$$
\left\{\lambda^{v}\right\}_{K} \rightarrow \bar{\lambda}=-\left[\nabla h\left(x^{*}\right)^{\top} \nabla h\left(x^{*}\right)\right]^{-1} \nabla h\left(x^{*}\right)^{\top}\left(\nabla \ell\left(x^{*}\right)+w^{*}\right) .
$$

Thus the active multipliers are bounded. As a result, (31) shows that the inactive multipliers will be zero. By defining the optimal multipliers using (27)-(28), the remainder of the proof is analogous to the proof of Theorem 13, with $I_{a}\left(x^{*}\right)$ replacing $I_{\bar{O}}\left(x^{*}\right)$ and of course with the exception of showing condition $(20 \mathrm{~g})$. This condition is however readily shown by recognizing that all multipliers $\Gamma_{i j}^{*}$ are nonpositive, so the product of two of them will always be nonnegative.

Remark 15 The result here mirrors the second special case of Theorem 3.2 in [9]. The authors consider an augmented Lagrangian method applied to the problem with complementarity constraints, and find that the assumption of the accumulation point being feasible and MPCC-LICQ holding, for unbounded penalty parameters, results in this point satisfying the Clarke stationarity conditions.

\section{Application: Model Predictive Control}

This section describes a Model Predictive Control (MPC) approach to autonomous navigation in an obstructed environment. The first subsection shows how the OCPs arising in MPC for trajectory control can be formulated according to problem (4). The second subsection presents simulation results showing the general applicability and efficiency of the proposed approach.

\subsection{Optimal control problem}

The MPC controller steers a mobile robot, also called the vehicle, from a starting point $\xi_{0}$ to a destination point $\xi_{\text {ref }}$, while avoiding $N_{O}$ obstacles in the environment. At every sampling time, this controller calculates the optimal sequence of $N$ control inputs, with $N$ denoting the length of the prediction horizon, by solving the following OCP

$$
\begin{aligned}
\underset{u \in \mathbb{R}^{N n_{u}}, \xi \in \mathbb{R}^{N \xi_{\xi}}}{\operatorname{minimize}} & \ell_{N}\left(\xi_{N}\right)+\sum_{k=0}^{N-1} \ell_{k}\left(\xi_{k}, u_{k}\right), \\
\text { subject to } & \xi_{k+1}=\varphi_{k}\left(\xi_{k}, u_{k}\right), k=0, \ldots, N-1, \\
& \psi_{j}\left(\xi_{k}\right)=0, j=1, \ldots, N_{O}, k=1, \ldots, N, \\
& u_{k} \in U_{k}, k=0, \ldots, N-1 .
\end{aligned}
$$

The input constraints can be grouped together in one set $U=U_{O} \times \ldots \times U_{N-1}$. Typically, these constraints represent actuator limits, which constrain the input to be between a minimum and a maximum value, $u_{\min } \leq u_{k} \leq u_{\max }$. The stage costs and the terminal cost express the distance of the state and input variables to the reference state and input. In MPC, they are typically quadratic functions of the form:

$$
\begin{aligned}
\ell_{k}\left(\xi_{k}, u_{k}\right) & =\left(\xi_{k}-\xi_{\mathrm{ref}}\right)^{\top} Q_{k}\left(\xi_{k}-\xi_{\mathrm{ref}}\right)+\left(u_{k}-u_{\mathrm{ref}}\right)^{\top} R_{k}\left(u_{k}-u_{\mathrm{ref}}\right), \\
\ell_{N}\left(\xi_{N}\right) & =\left(\xi_{N}-\xi_{\mathrm{ref}}\right)^{\top} Q_{N}\left(\xi_{N}-\xi_{\mathrm{ref}}\right) .
\end{aligned}
$$


Here, $Q_{k}, R_{k}$ and $Q_{N}$ are positive definite matrices. The system dynamics $\varphi_{k}$ represent the state evolution starting from the state at time $k$ under the application of control input $u_{k}$. Obstacles in this formulation are considered static, but dynamic obstacles may be incorporated as well, making the obstacle cost function additionally dependent on $k$.

To retrieve the formulation analyzed in this paper three steps are necessary. First, the multiple-shooting (MS) formulation of (32) with states and inputs as decision variables can be transformed to a single-shooting (SS) formulation with only the inputs as decision variables. For this, the states are written as functions of the input sequence $u=\left(u_{0}, \ldots, u_{N-1}\right)$ using the system dynamics, with $\xi_{0}=\Phi_{0}(u)$ as a parameter,

$$
\xi_{k+1}=\Phi_{k+1}(u)=\varphi_{k}\left(\Phi_{k}(u), u_{k}\right) .
$$

Second, the objective function can be defined as $\ell(u)=$ $\ell_{N}\left(\Phi_{N}(u)\right)+\sum_{k=0}^{N-1} \ell_{k}\left(\Phi_{k}(u), u_{k}\right)$. Finally, the obstacle cost functions $\psi_{j}\left(\Phi_{k}(u)\right)$ now apply to the dynamics instead of directly to the decision variables. These functions can still be considered obstacles by including the dynamics in the obstacle boundary functions as follows

$$
\Phi_{k}(u) \in O_{j} \Longleftrightarrow u \in O_{j k},
$$

with $O_{j k}=\left\{u \in \mathbb{R}^{N n_{u}}: h_{j l}\left(\Phi_{k}(u)\right)>0, l=1, \ldots, m_{j}\right\}$. As $\Phi_{k}(u)$ are smooth functions, the chain rule can be applied for calculating the derivatives of the obstacle boundary functions. Hence problem (32) can readily be written as a problem of type (4), and Theorems 13 and 14 apply, given we use a suitable solver for the inner subproblems. In this work, we employ the proximal averaged Newton-type method for optimal control, PANOC [20] as the inner solver, as it can provide a point satisfying (8) and exhibits fast convergence properties while being light-weight and limited in memory usage. The method here is therefore fairly scalable, although our main focus is relatively small OCP.

\subsection{Simulation results}

An implementation of the proposed method in $\mathrm{C}$ is versatile in modeling complex obstacle shapes and efficiently calculating trajectories for a kinematic bicycle model. The bicycle $[15$, p. 26$]$ is a system with four states, $\xi=\left(q_{1}, q_{2}, v, \theta\right)$, a horizontal position, a vertical position, a velocity and a heading angle. It is controlled via two inputs, $u=(a, \delta)$, the acceleration and the steering angle of the front wheel. The continuous kinematics relating $u$ to $\dot{\xi}$ are given by

$$
\begin{aligned}
\dot{q}_{1} & =v \cdot \cos (\theta+\beta), & \dot{q}_{2} & =v \cdot \sin (\theta+\beta), \\
\dot{\theta} & =\frac{v}{l_{r}} \sin (\beta), & \dot{v} & =a,
\end{aligned}
$$

where the slip angle $\beta=\tan ^{-1}\left(\frac{l_{r}}{l_{r}+l_{f}} \tan (\delta)\right)$, and $l_{r}=1.17 \mathrm{~m}$ and $l_{f}=1.77 \mathrm{~m}$ are the distances from the center to the rear and front wheel respectively. The kinematics were discretized using an explicit fourth order Runge Kutta method.
Figure 1 illustrates how two nonconvex obstacles forming an S-shaped lane, given by $O_{1}=\left\{\left(q_{1}, q_{2}\right): 0<q_{1}<5,-2<\right.$ $\left.q_{2}<2+\frac{3}{2} \sin \left(\frac{2 \pi}{5} q_{1}\right)\right\}$ and $O_{2}=\left\{\left(q_{1}, q_{2}\right): 0<q_{1}<5,4+\right.$ $\left.\frac{3}{2} \sin \left(\frac{2 \pi}{5} q_{1}\right)<q_{2}<8\right\}$, are successfully avoided by the MPCcontrolled bicycle. Here, the sampling time $t_{s}=50 \mathrm{~ms}$, and a horizon length $N=30$. The input acceleration and steering angle were constrained by box constraints $-10 \mathrm{~m} / \mathrm{s}^{2} \leq a \leq$ $10 \mathrm{~m} / \mathrm{s}^{2}$ and $-\frac{\pi}{4} \mathrm{rad} \leq \delta \leq \frac{\pi}{4} \mathrm{rad}$ at every time instant. The parameters used in Algorithm 1 are: $x^{0}=u^{0}=0, \mu^{1}=100$, $\omega=10, \varepsilon^{0}=1, \varepsilon^{v+1}=10^{-1} \varepsilon^{v}, \varepsilon^{*}=10^{-3}$ and $\eta^{*}=10^{-2}$.

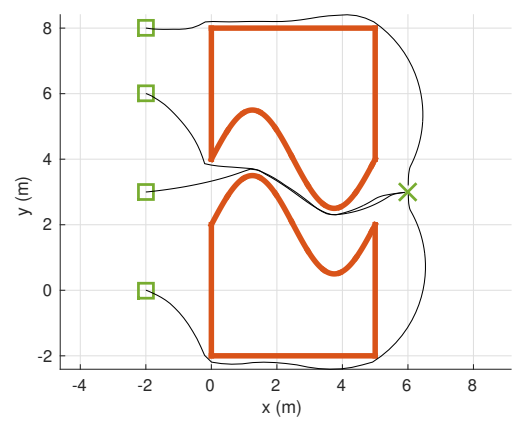

Fig. 1. The MPC-controller steers the vehicle from different starting points (green squares) to the destination (green cross) in the presence of two obstacles (red) forming an S-shaped lane.

Table 1 shows a comparison in terms of runtime and objective value of the proposed quadratic penalty method (QPM) with solvers that solve the equivalent problem with complementarity constraints (10), for 50 instances of the OCP, each with a random initial state. In the case of SNOPT and IPOPT, the complementarity constraints were replaced by their NLP version $t_{i j} \geq 0, \forall i, j$ and $\sum_{i=1}^{n} \prod_{j=1}^{m_{j}} t_{i j} \leq 0$. Aside from our method relying on PANOC, which has no inherent capability to deal with equality constraints, the solvers were tested for both an SS and an MS formulation. All simulations were performed on a notebook with Intel(R) Core(TM) i7-7600U CPU @ 2.80GHz x 2 processor and 16 GB of memory. For all simulations, the automatic differentiation package CasADi [1] was used to efficiently evaluate the objective and constraint functions and gradients.

Fail percentage indicates the frequency of converging to something other than an approximate local minimum. No time was measured during problem construction, and only the reported runtimes by the solvers are presented. The performance in terms of objective values for each solver $s_{i}$ is reported as the relative difference between the objective value of the optimal input sequence for that solver $u^{*, s_{i}}$, and the best solution found by all solvers for each instance, i.e. $\frac{f\left(u^{*, s_{i}}\right)-f^{*}}{f^{*}}$, with $f^{*}=\min _{s \in S}\left\{u^{*, s}\right\}$, with $S$ the set of all solvers. This measure indicates the quality of the solution, which is interesting as problems with set exclusion constraints are highly nonconvex and therefore many local minima exist. Typically, because of the large terminal cost, trajectories where the vehicle remains in place behind an obstacle are local minima with a higher objective value than 
trajectories that find their way to the destination. Hence, the proposed method, which exhibits the lowest average and maximum differences, is shown to be effective at finding meaningful local minima for these types of path planning problems. Furthermore, in terms of runtime and success percentage, the proposed method is also shown to outperform all other methods.

Table 1

Performance comparison for an MPC controlled bicycle.

\begin{tabular}{l|ccccc}
\hline \multirow{2}{*}{ Solver } & \multicolumn{2}{|c}{ Runtime (s) } & \multicolumn{2}{c}{$\frac{f\left(u^{*, s_{i}}\right)-f^{*}}{f^{*}}$} & \multirow{2}{*}{ Fail (\%) } \\
& Avg & Max & Avg & Max & \\
\hline QPM (SS) & 0.017 & 0.115 & 0.19 & 1.12 & 8 \\
IPOPT (SS) & 1.731 & 7.776 & 0.25 & 1.24 & 14 \\
IPOPT (MS) & 1.604 & 4.448 & 0.38 & 8.55 & 16 \\
SNOPT (SS) & 9.320 & 4.335 & 0.29 & 2.02 & 16 \\
SNOPT (MS) & 1.153 & 3.054 & 0.42 & 1.43 & 78 \\
KNITRO (SS) & 4.391 & 13.73 & 0.64 & 10.9 & 38 \\
KNITRO (MS) & 14.13 & 19.78 & 0.60 & 1.42 & 74 \\
\hline
\end{tabular}

\section{Conclusion}

This paper presented theoretical convergence results for optimization problems with general nonsmooth set exclusion constraints. These problems were solved by embedding the exclusion constraints into a quadratic penalty framework, and solving the inner subproblems using PANOC. The limit points of the generated sequences were shown to satisfy either the strong stationarity conditions or the Clarke stationarity conditions of a reformulated problem with complementarity constraints, depending on the assumptions made. Furthermore, numerical simulations of MPC applied to a kinematic bicycle illustrate that the proposed solution method is versatile and outperforms other solvers applied to the equivalent problem with complementarity constraints.

\section{Acknowledgements}

This work benefits from KU Leuven-BOF PFV/10/002 Centre of Excellence: Optimization in Engineering (OPTEC), from the project G0C4515N of the Research Foundation-Flanders (FWOFlanders), from Flanders Make ICON project: Avoidance of collisions and obstacles in narrow lanes, and from the KU Leuven Research project C14/15/067: B-spline based certificates of positivity with applications in engineering, from KU Leuven internal funding: StG/15/043, from Fonds de la Recherche Scientifique FNRS and the Fonds Wetenschappelijk Onderzoek - Vlaanderen under EOS Project no 30468160 (SeLMA) and from FWO projects G086318N, G086518N.

\section{References}

[1] Joel A E Andersson, Joris Gillis, Greg Horn, James B Rawlings, and Moritz Diehl. CasADi - A software framework for nonlinear optimization and optimal control. Mathematical Programming Computation, In Press, 2018.

[2] Mihai Anitescu. Global convergence of an elastic mode approach for a class of mathematical programs with complementarity constraints. SIAM Journal on Optimization, 16(1):120-145, 2005.

[3] Dimitri P Bertsekas. Constrained optimization and Lagrange multiplier methods. Academic press, 1982.

[4] Ernesto G Birgin and Josâ Mario Martınez. Practical augmented Lagrangian methods for constrained optimization, volume 10. SIAM, 2014.

[5] Frank H Clarke. A new approach to Lagrange multipliers. Mathematics of Operations Research, 1(2):165-174, 1976.

[6] Elmer Gilbert and Daniel Johnson. Distance functions and their application to robot path planning in the presence of obstacles. IEEE Journal on Robotics and Automation, 1(1):2130, 1985.

[7] Philip E Gill, Walter Murray, and Michael A Saunders. SNOPT: An SQP algorithm for large-scale constrained optimization. SIAM review, 47(1):99-131, 2005.

[8] Ben Hermans, Panos Patrinos, and Goele Pipeleers. A penalty method based approach for autonomous navigation using nonlinear model predictive control. In 6th IFAC Conference on Nonlinear Model Predictive Control NMPC 2018, pages 268-274, 2018.

[9] Alexey F Izmailov, Mikhail V Solodov, and E I Uskov. Global convergence of augmented lagrangian methods applied to optimization problems with degenerate constraints, including problems with complementarity constraints. SIAM Journal on Optimization, 22(4):1579-1606, 2012.

[10] Michael L Flegel. Constraint qualifications and stationarity concepts for mathematical programs with equilibrium constraints /. 042005

[11] Sven Leyffer. Complementarity constraints as nonlinear equations: Theory and numerical experience. In Optimization with Multivalued Mappings, pages 169-208. Springer, 2006.

[12] Sven Leyffer, Gabriel López-Calva, and Jorge Nocedal. Interior methods for mathematical programs with complementarity constraints. SIAM Journal on Optimization, 17(1):52-77, 2006.

[13] Tim Mercy, Ruben Van Parys, and Goele Pipeleers. Splinebased motion planning for autonomous guided vehicles in a dynamic environment. IEEE Transactions on Control Systems Technology, 2017.

[14] Rushen B Patel and Paul J Goulart. Trajectory generation for aircraft avoidance maneuvers using online optimization. Journal of guidance, control, and dynamics, 34(1):218-230, 2011.

[15] Rajesh Rajamani. Vehicle dynamics and control. Springer Science \& Business Media, 2011.

[16] R Tyrrell Rockafellar and Roger J-B Wets. Variational analysis, volume 317. Springer Science \& Business Media, 2009.

[17] Ajay Suresha Sathya, Pantelis Sopasakis, Ruben Van Parys, Andreas Themelis, Goele Pipeleers, and Panos Patrinos. Embedded nonlinear model predictive control for obstacle avoidance using PANOC. In Proceedings of the 2018 European Control Conference, 2018.

[18] Holger Scheel and Stefan Scholtes. Mathematical programs with complementarity constraints: Stationarity, optimality, and sensitivity. Mathematics of Operations Research, 25(1):1-22, 2000.

[19] Stefan Scholtes and Michael Stöhr. How stringent is the linear independence assumption for mathematical programs with 
complementarity constraints? Mathematics of Operations Research, 26(4):851-863, 2001.

[20] Lorenzo Stella, Andreas Themelis, Pantelis Sopasakis, and Panagiotis Patrinos. A simple and efficient algorithm for nonlinear model predictive control. In 56th IEEE Conference on Decision and Control, pages 1939-1944, 2017.

[21] Richard W Cottle and George B Dantzig. A generalization of the linear complementarity problem. Journal of Combinatorial Theory, 2:79-90, 011970.

[22] Andreas Wächter and Lorenz T Biegler. On the implementation of an interior-point filter line-search algorithm for largescale nonlinear programming. Mathematical programming, 106(1):25-57, 2006.

[23] Richard A Waltz and Jorge Nocedal. KNITRO user's manual. Northwestern University, Evanston, Illinois, Technical Report OTC-2003/5, 2003.

[24] Peng Wang and Baocang Ding. A synthesis approach of distributed model predictive control for homogeneous multiagent system with collision avoidance. International Journal of Control, 87(1):52-63, 2014. 